\title{
A Note on the Diophantine Equation
}

$$
x^{n}+y^{n}+z^{n}=3
$$

\section{By Manny Scarowsky and Abraham Boyarsky*}

\begin{abstract}
In this note solutions for the Diophantine equation

$$
x^{3}+y^{3}+z^{3}=3
$$

are sought along planes $x+y+z=3 m, m \in \mathbf{Z}$. This was done for $|m| \leqslant 50000$, and no new solutions were found.
\end{abstract}

1. Introduction. The existence of integral solutions of

$$
x^{3}+y^{3}+z^{3}=3
$$

has been investigated in [1, pp. 100-101]. For the range of $0 \leqslant x \leqslant y \leqslant 65,536$, $0<-z-x<65,536$, or $0 \leqslant-x \leqslant-y \leqslant 65,536,0<z+x<65,536$ or for the range $\max (|x|,|y|,|z|) \leqslant 3164$, the only known solutions are $(1,1,1)$ and $(4,4,-5)$ and its permutations. We note by using Fermat's Little Theorem that $x+y+z$ is divisible by 3 . This suggests that instead of searching for solutions blindly, we parametrize the search by $x+y+z=3 m, m \in \mathbf{Z}$. This was done for $|m| \leqslant 50000$ without finding solutions other than the two aforementioned ones.

We also consider briefly the equation $x^{n}+y^{n}+z^{n}=3$ with the same parametrization and show that for each $m$ there can only be a finite number of (effectively determinable) solutions, and that the irreducible polynomial $f_{n}(m)=3^{n-1} m^{n}-1$ must have at least four prime factors for each $m$, except for a finite number of (effective) exceptions.

2. Results. Assume that $x^{n}+y^{n}+z^{n}=3, n$ odd and greater than $1, x, y, z \in \mathbf{Z}$. It follows that $x+y+z=3 m$ for some $m \in \mathbf{Z}$.

Lemma 1. Let $m$ be an integer. Then there exist at most finitely many (effectively computable) triples of integers $(x, y, z)$ such that

$$
x^{n}+y^{n}+z^{n}=3, \quad x+y+z=3 m .
$$

Proof. It is known that if $X, Y, Z$ are indeterminates, then

$$
(X+Y+Z)^{n}-\left(X^{n}+Y^{n}+Z^{n}\right)=(X+Y) \cdot(Y+Z)(Z+X) Q(X, Y, Z),
$$

where $Q(X, Y, Z)$ is a polynomial with integral coefficients. If $(x, y, z)$ is a triplet satisfying the system (A), then $(3 m)^{n}-3=(x+y)(y+z)(x+z) Q(x, y, z)$. Hence

Received Scptember 21, 1982.

1980 Mathematics Subject Classification. Primary 10B 15

*The research of this auihor was supported by a grant from the National Research Council of Canada, (irant No. A-9072. 
$x+y, y+z, z+x$ belong to the finite set of factors of $(3 m)^{n}-3$. Thus $x, y, z$ belong to a finite set which is effectively computable.

LEMma 2. Given $n \geqslant 3, n$ odd, then there exists an effective $C>0$ such that if $x, y, z$ and $m$ are integers satisfying $(\mathrm{A})$ and if, moreover, $3^{n-1} m-1=p_{1}, p_{1} p_{2}$, or $p_{1} p_{2} p_{3}$ (where each $p_{i}$ is a prime), then $|x|,|y|,|z|,|m|<C$.

Proof. Assume that $f_{n}(m)=3^{n-1} m^{n}-1$ has less than 4 prime factors. As in Lemma 1,

$$
f_{n}(m)=\frac{1}{3}(x+y)(x+z)(y+z) Q(x, y, z) .
$$

If $f_{n}(m)=p_{1}$ or $p_{1} p_{2}$, then (possibly by renaming $\left.x, y, z\right)$ we must have $x+y= \pm 1$, \pm 3 . If $f_{n}(m)=p_{1} p_{2} p_{3}$, then again $x+y= \pm 1, \pm 3$ or one of the $p_{i}$ is even $(=2)$ and $x+y= \pm 2, \pm 6$. It is well known that the system $x^{n}+y^{n}+z^{n}=3, x+y=a$. where $a \in\{ \pm 1, \pm 2, \pm 3, \pm 6\}$ has a finite number of solutions which in principle can be effectively determined [2]. This completes the proof.

Remarks. (1) Lemma 2 suggests that the search for solutions of $x^{n}+y^{n}+z^{n}=3$ be made among $m$ such that $f_{n}(m)$ has many prime factors.

(2) Lemma 2 can be generalized to the equation $x^{n}+y^{n}+z^{n}=3 d$.

We end this section with an open problem: Does there exist an odd $n \geqslant 3$ such that $x^{n}+y^{n}+z^{n}=3$ has only a finite number of solutions? Note that both the equations $x^{3}+y^{3}+z^{3}=1, x^{3}+y^{3}+z^{3}=2$ have an infinite number of solutions $[1$, p. 100].

3. Numerical Results. A numerical search for solutions of $x^{3}+y^{3}+z^{3}=3$ was made using the parametrization $x+y+z=3 m$, where $m$ is odd. Since

$$
f_{3}(m)=9 m^{3}-1=(x+y)(y+z)(x+z),
$$

we can assume that one of the factors, say $x+y$, of $9 m^{3}-1$ satisfies:

$$
|x+y| \leqslant\left|\left(9 m^{3}-1\right)^{1 / 3}\right|=M .
$$

For each value of $m$, we let $x+y$ run from $-M$ to $M$ in steps of $3(x+y \equiv 2(\bmod$ 3); see Remark 2 below), testing for each value of $x+y$ if it divides $9 m^{3}-1$. If it does, we solve for $x, y$, using the values: $A=x+y$,

$$
\begin{aligned}
& B=\frac{9 m^{3}-1}{x+y}=x y+3 m(3 m-A), \\
& x=\frac{1}{2}\left(A \pm \sqrt{A^{2}-4 B+12 m(3 m-A)}\right), \\
& y=\frac{1}{2}\left(A \mp \sqrt{A^{2}-4 B+12 m(3 m-A)}\right) .
\end{aligned}
$$

Now, $x, y$ and $z=3 m-x-y$ satisfy $x^{3}+y^{3}+z^{3}=3$ if and only if the square root term in (1) is an integer.

The search was done on the CDC CYBER 170 using double precision. In the time allotted for this project (18 hours CPU time), we were able to let $m$ range from $-50,000$ to $+50,000$. No new solutions were found. As an example of the computer times involved, it took $2760 \mathrm{CPU}$ seconds for the program to run from $m=44,945$ to $m=46,965$. Double precision on the CDC CYBER 170 allows 29 significant digits. For $-50,000 \leqslant m<50,000$, the quantity $C=A^{2}-4 B+12 m(3 m-A)$ is 
at most of the order $10^{16}$. Now let $\sqrt{C}$ denote the true square root of $C$ and $\operatorname{sqr}(C)$ the computed value of the square root of $C$. To test if $\sqrt{C}$ is an integer, we computed

$$
z=\operatorname{int}[\operatorname{sqr}(C)+.25]
$$

$z$ is always an integer and the nearest integer to $\operatorname{sqr}(C)$. To test if $z=\sqrt{C}$, we check if $C=z^{2}$. If so, $z=\sqrt{C}$, otherwise it is not. For example, if $C=168$, then $z=13$ and $z^{2}=169 \neq C$.

It is important to note that for this test to be effective, it is necessary that $|\sqrt{ } C-\operatorname{sqr}(C)| \leqslant .25$. Since $C$ is of the order of $10^{16}$ and the computer accuracy is up to 29 digits, this will always be the case.

Remarks. (1) It is easy to see that $x^{3}+y^{3}=3-z^{3}$ has no solutions if $3-z^{3}=$ $\pm 3^{j} \Pi p_{i}^{a_{i}}$, where $j \geqslant 0, a_{i} \geqslant 0$, and all the $p_{i}$ are distinct primes of the form $p_{i} \equiv 5$ $(\bmod 6)$.

(2) For $x^{3}+y^{3}+z^{3}=3$ it can be seen that 3 is a cubic residue of every prime factor of $x^{3}+y^{3}, y^{3}+z^{3}, x^{3}+z^{3}$. (Note, for example, with regard to the solution $(4,4,-5)$, we have $(3 / 61)_{3}=1$.) This fact can possibly be used to speed up the search for solutions in larger domains. Also, it is easy to show that in order to have solutions of $x^{3}+y^{3}+z^{3}=3$ we must have $x \equiv y \equiv z \equiv 1(\bmod 3)$. This was used to speed up the search.

(3) For $m=50,000, x+y+z=150,000$. Hence $0<x+z<150,000$, which clearly far exceeds the range of values $0<x+z<65,536$ in the previous blind search. Also the second range searched, $\max (|x|,|y|,|z|) \leqslant 3164$, is far exceeded.

Acknowledgement. The authors are grateful to the referee for his comments and to Bert Buckley for helpful discussions.

Department of Mathematics

Loyola Campus

Concordia University

Montreal, Quebec, Canada H4B IR6

I. L. J. Mordell., Diophantine Equations, Academic Press, New York, 1969.

2. A. Baker, Transcendental Number Theory, Cambridge Univ. Press, Cambridge, 1975. 